\title{
PHENOTYPIC AND GENOTYPIC IDENTIFICATION OF CHITIN PRODUCING FUNGI AND STUDY OF THEIR PHARMACEUTICAL APPLICATION
}

\section{Muddukrishnaiah K*, Shilpa V P, Samuel Thavamani B, Jayaprakash G, Dhanapal V \\ Department of Pharmaceutical Biotechnology, Sanjo College of Pharmaceutical studies, Kerala University of Health Science, Vellapara, Palakkad, India}

\section{ABSTRACT}

Objectives: Current days, the production and utilization of natural biodegradable polymers have wide application in pharmaceutical and biological fields. The aim of the present study is to isolate and identify chitin polymer from Aspergillus japonicas Sanjo College of Pharmaceutical Studies (SCOPS-A1) and to study their role in pharmaceutical applications. Methods: Aspergillus japonicas SCOPS - A1 was isolated form Phyllanthus niruri plant leaf from Palakkad, Kerala and it was identified by fungal universal primers ITS4 \& ITS5 at ITS region of rDNA (NCBI Accession numbers MG833000, MG833001). The chitin was isolated from Aspergillus japonicas SCOPS A1 by submerged culture using potato dextrose broth. $200 \mathrm{mg} / \mathrm{g}-1$ (milligrams of chitin polymer/gram of dry biomass) of chitin polymer was extracted from 15-days mycelia by alkali - acid treatment. Extracted Chitin was characterized by Fourier transform infrared spectroscopy (FTIR), and X-Ray Diffraction (XRD). Chitin-Almotriptan malate microspheres were prepared by water in-oil-in-oil double emulsion method. Results: To highlight Chitin pharmaceutical application, Chitin-Almotriptan malate microspheres were prepared by water-in-oil-in-oil double emulsion method which is used for migraine treatment. Infrared spectroscopy analysis conform the absence of drug and polymer interaction.Conclusion: In this study we conclude that Aspergillus japonicas SCOPS - A1 produces Chitin and it can be used as the choice of polymer for Pharmaceutical formulation.

Keywords: Aspergillus japonicas SCOPS - A1, Chitin, Microspheres, FTIR.

\section{INTRODUCTION}

Aspergillus species are saprophytic, ubiquitous fungi that can be found worldwide, mainly present in plants, water, soil, air and food. Chitin is a polymer of $\mathrm{N}$ acetyl-D-glucosamine monomer units having $\beta-1-4$ glycosidic linkage, most copious renewable natural resources. Chitin mainly present in crustaceans and also the main cell wall component in fungi. Chitin is a non-toxic, biodegradable, biocompatible and mucoadhesive natural polymer. Developing a better drug delivery system will decrease pharmacological toxicity, enhance sensitivity and specificity of the drug. Microspheres are carrier mediated drug delivery formulation which acts as an important role in novel drug delivery system. Microspheres are free flowing, spherical and monolithic matrix type component. [1] Almotriptan is a second-generation 5-HT (1B/1D) agonist that exhibits vascular selectivity for meningeal arteries and has efficacy for the treatment of acute migraine. Almotriptan notably reduces blood flow through extra cerebral cranial vessels and increases cerebral blood flow.

Figure 1 Different type of fungi growth on Phyllanthus niruri plant extract surface

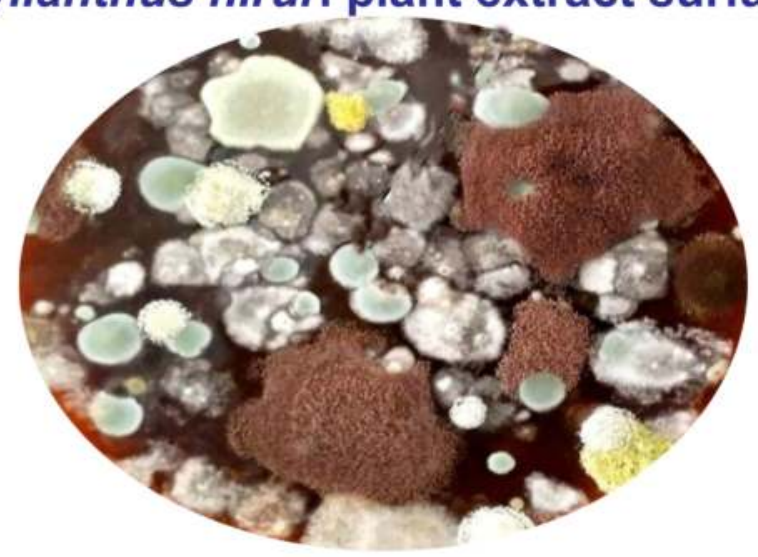

RESULTS AND DISCUSSION

Morphological and Molecular Identification
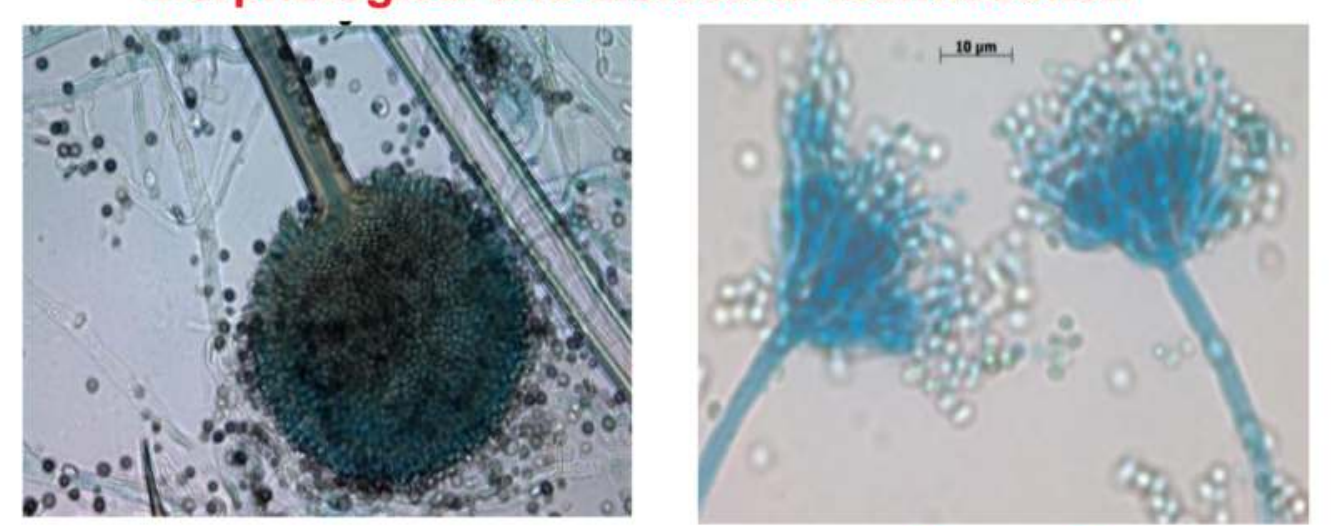

Figure 2
Molecular Identification

Table 1 Top five hits upon BLASTn analysis

\begin{tabular}{|l|l|c|c|c|c|c|}
\hline $\begin{array}{c}\text { Gene Bank } \\
\text { Accession No. }\end{array}$ & \multicolumn{1}{|c|}{ Description } & $\begin{array}{c}\text { Max } \\
\text { score }\end{array}$ & $\begin{array}{c}\text { Query } \\
\text { cover }\end{array}$ & $\begin{array}{c}\text { Query } \\
\text { coverage }\end{array}$ & $\begin{array}{c}\text { E } \\
\text { value }\end{array}$ & $\begin{array}{c}\text { Identity } \\
(\%)\end{array}$ \\
\hline KY8486666.1 & Aspergillus japonicus isolate SF1 & 933 & 933 & $100 \%$ & 0.0 & $100 \%$ \\
\hline KY086235.1 & Aspergilllus sp. isolate JSM 06261661 & 933 & 933 & $100 \%$ & 0.0 & $100 \%$ \\
\hline KX011594.1 & Aspergillus aculeatus strain IM31 & 933 & 933 & $100 \%$ & 0.0 & $100 \%$ \\
\hline KJ439214.1 & Aspergillus japonicus isolate V42-355 & 933 & 933 & $100 \%$ & 0.0 & $100 \%$ \\
\hline KJ192202.1 & Aspergillus cf. japonicus C15-68 & 933 & 933 & $100 \%$ & 0.0 & $100 \%$ \\
\hline
\end{tabular}

\section{Growth kinetics}

Isolation and characterization

Figure 4 Growth kinetics of Aspergillus japonicas SCOPS - A1 Figure 5 FTIR Spectrum

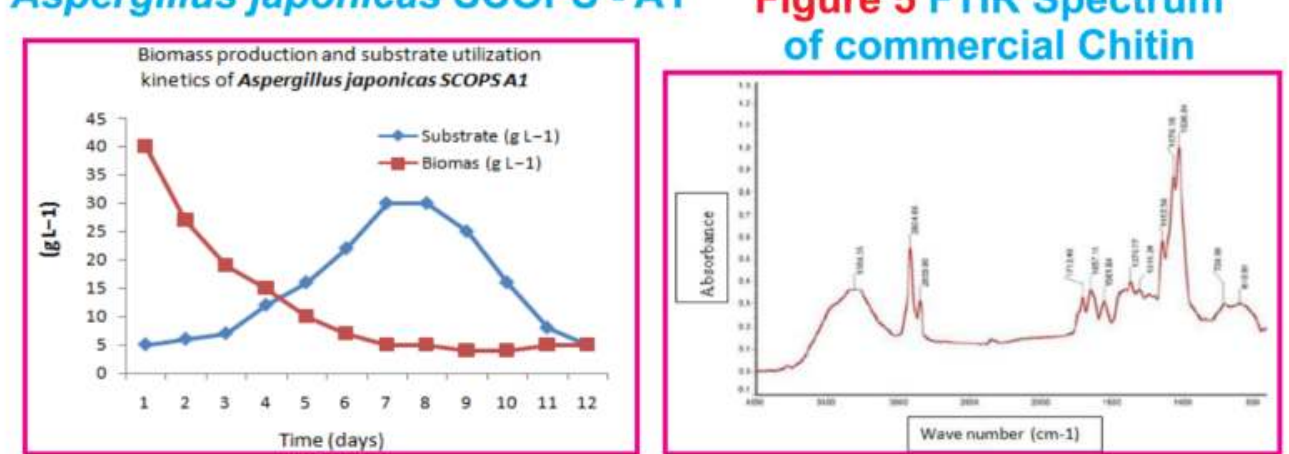

Figure 6 FTIR Spectrum of Chitin xtracted from Aspergillus japonicas SCOPS - A1

Table 2 : Calibration Curve of AlmotriptanMalate

\begin{tabular}{|c|c|}
\hline Concentration $(\mu \mathrm{g} / \mathrm{ml})$ & Absorbance $(\mathrm{nm})$ \\
\hline 0 & 0 \\
\hline 2 & 0.2166 \\
\hline 4 & 0.4085 \\
\hline 6 & 0.6155 \\
\hline 8 & 0.8098 \\
\hline 10 & 1.0031 \\
\hline
\end{tabular}

Compatibility Studies of drug \& excipients Figure 8 FTIR of Almotriptan Malate
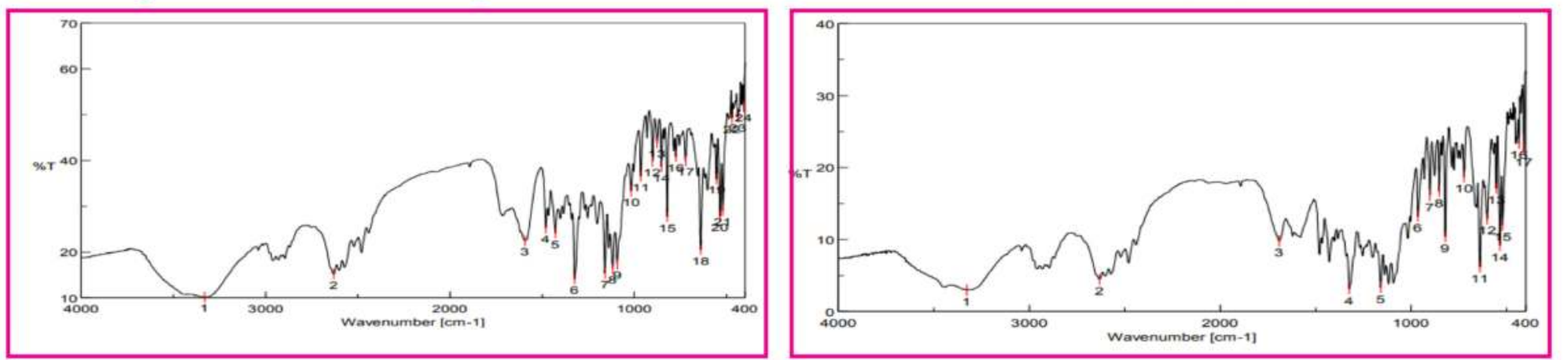

Microspheres

Figure 10 Almotryptan maleate +

Chitin (polymer) microspheres (SEM)

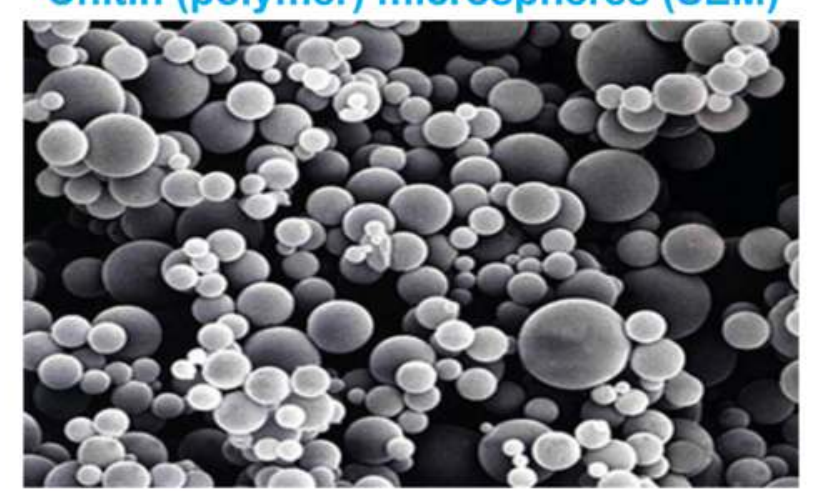

.In-vitro study of Chitin microspheres for Almotriptan maleate drug delivery Table 3 Determination of In-vitro release profile

\begin{tabular}{|c|c|c|}
\hline Time(hr) & F1 & F2 \\
\hline 1 & 11.88 & 19.93 \\
\hline 2 & 15.66 & 22.15 \\
\hline 3 & 18.9 & 26.58 \\
\hline 4 & 20.52 & 31.01 \\
\hline 5 & 26.96 & 38.76 \\
\hline 6 & 35.64 & 49.29 \\
\hline 7 & 46.44 & 61.47 \\
\hline 8 & 58.86 & 75.32 \\
\hline
\end{tabular}

Figure 11 In-vitro studies of Chitin microspheres for Almotriptan maleate

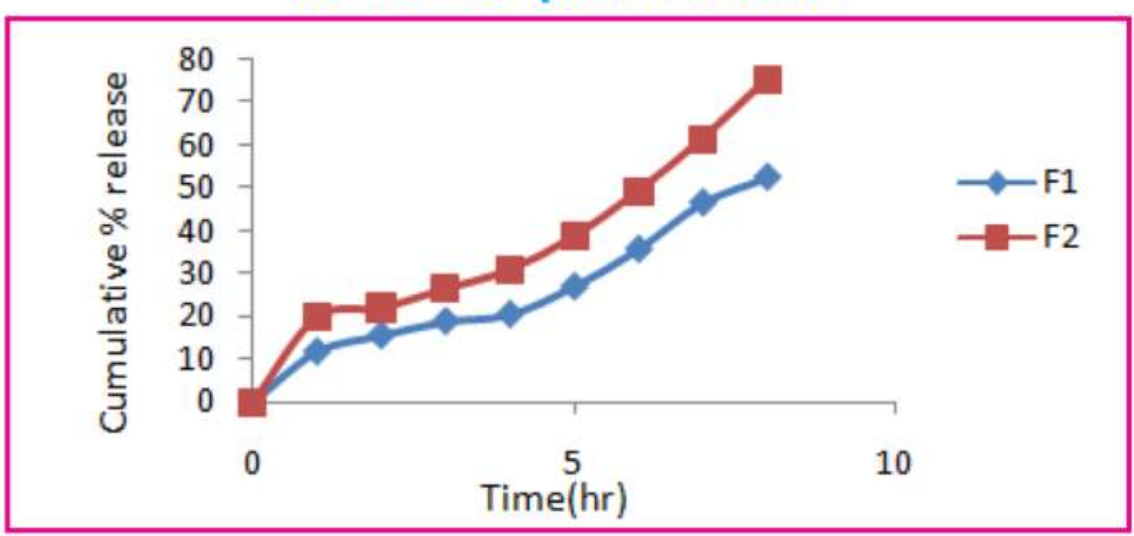

Drug release kinetics of Microspheres formulation Figure 12 Drug release kinetics

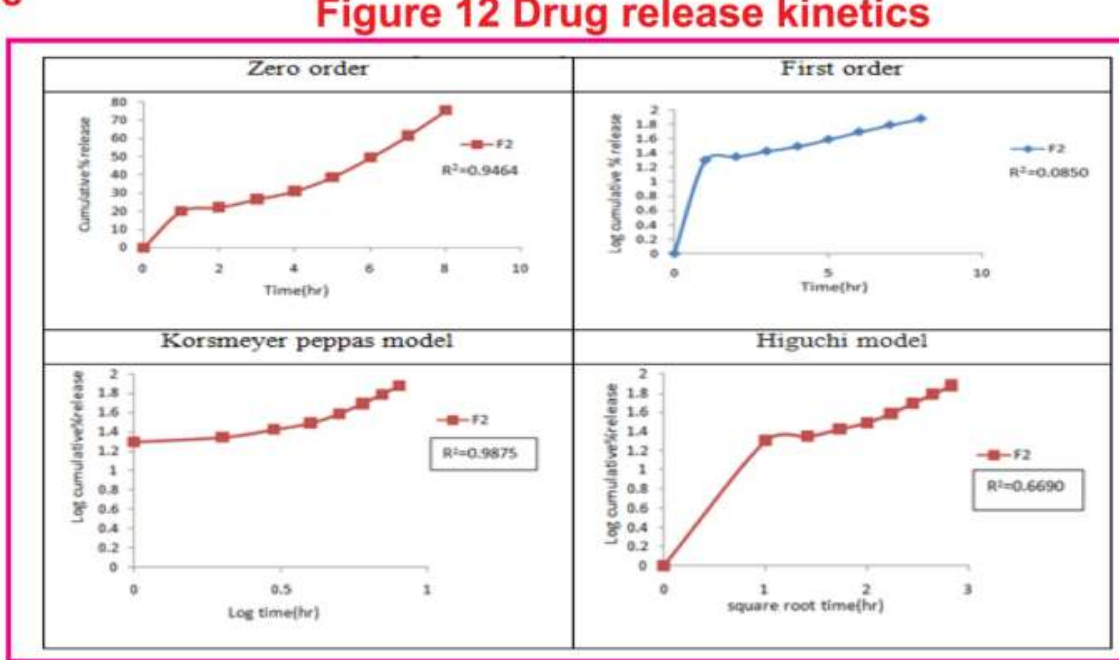

\section{DISCUSSION}

Almotriptan malate is a typical second-generation 5-HT (1B/1D) agonist mainly used in the treatment of migraine. In order to increase the drug release, an attempt was made to formulate Almotriptan malate in the form of microspheres. Aspergillus japonicas SCOPS - A1 Chitin was used as polymer blend for the preparation of microspheres. Based on physicochemical parameters and in-vitro release studies, formulation F2 with Chitin polymer was considered to be the best formulation.

\section{REFERENCES}

1.Yang Qu, Jinyu Xu, Haohan Zhou, Rongpeng Dong, Mingyang Kang and Jianwu Zhao: Chitin Oligosaccharide (COS) Reduces Antibiotics Dose and Prevents Antibiotics-Caused Side Effects in Adolescent Idiopathic Scoliosis (AIS) Patients with Spinal Fusion Surgery. Mar. Drugs, 15, 70, 2017.

2.Michelle L. Ratay, Stephen C. Balmert, Abhinav P. Acharya, Ashlee C. Greene, Thiagarajan Meyyappan \& Steven R. Little: TR Microspheres prevent key signs of dry eye disease in a murine, inflammatory model. Nature scientific reports 7: 17527, 2017. 